\title{
Computed Tomography of a Lymph Node Basin with Contrast
}

National Cancer Institute

\section{Source}

National Cancer Institute. Computed Tomography of a Lymph Node Basin with Contrast. NCl Thesaurus. Code C137890.

Computed tomography of one or more lymphatic basins with the use of a contrast agent to enhance the image. 